\title{
„Get connected!“
}

lautet das diesjährige Motto von BIO-RAD auf der MEDICA Düsseldorf, 17.-20.11.1999.

Mit dem zukunftsorientierten Labor-Network-Systemen CSN und Unity kommunizieren BIO-RADKunden weltweit.

Durch den Vergleich mit über 10.000 Labors sind sowohl Problemlösung, sofortiger Informationsaustausch und die schnelle, sichere Kontrollergebnis-Prüfung gewährleistet.

\section{Weitere Schwerpunkte von BIO-RAD sind:}

- Vollautomatische Hämoglobin-Testsysteme

- Komplettlösung für die Klinische HPLC.

Seit über 30 Jahren produziert und vertreibt BIO-RAD Geräte und Reagenzien zur Bestimmung wichtiger klinischer Analyte.

Weitere Informationen erhalten Sie am Stand $3 A 72$

„Get connected!““

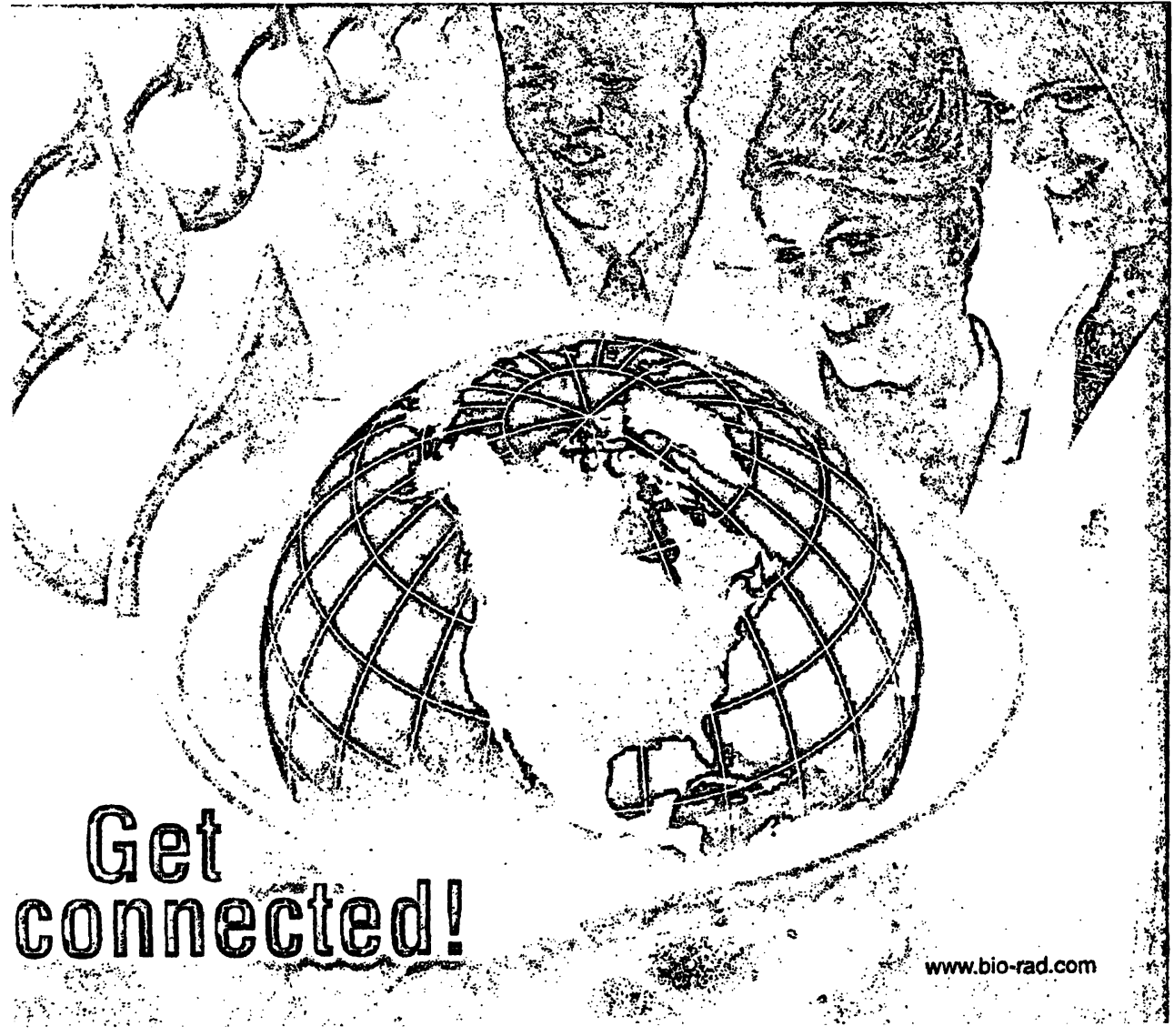

Mit den zukunftsorientie klinischen Labor-Netzwer Systemen CSN und Unity kommunizieren Bio-Rad Kunden weltweit!

- Problemlösung und Informationsaustausch

a Schnelle und sichere Kontrollergebnis-Prüfu durch den Vergleich mit über 10.000 Labors

Besuchen Sie uns auf deI Medica Düsseldorf 17. - 20. Nov. 1999 Halle 3, Stand $3 A 72$ und informieren Sie sich auch über

- Qualitätskontrollmanagement für Ihre Sicherheit

- Vollautomatische Hämoglobin $\mathrm{HbA}_{1 \mathrm{c}}$ Test-Systeme

$\square$ Komplettlösungen für die Klinische HPLC

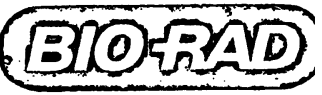

Bio-Rad Laboratories $\mathrm{GmbH}$ Heidemannstraße 164 D-80939 München 


\section{Zahl der AIDS-Neuerkrankungen geht zurück, aber Zahl der HIV-Infektionen bleibt unvermindert hoch}

Auf Grund der in den letzten Jahren erheblich verbesserten Therapiemöglichkeiten ist die Zahl der neu mit einem AIDS-Vollbild diagnostizierten Patienten deutlich zurückgegangen. Unter Berücksichtigung des Meldeverzugs, d.h. der Korrektur für noch zu erwartende Fallmeldungen, wird die Zahl der 1998 neu mit dem AIDS-Vollbild diagnostizierten Patienten auf etwa 800 zurückgehen. Im Vergleich zu den Jahren 1993/1994, in denen mit ca. 1900 die bisher höchste Zahl an Erkrankten gemeldet wurde, bedeutet dies einen Rückgang der AIDS-Neudiagnosen um mehr als 50\%.

Ebenso ist die Zahl der Todesfälle, die auf eine HIV-Infektion bzw.AIDS zurückzuführen sind, in Deutschland stark zurückgegangen. Starben 1994 noch über 2100 Menschen an einer HIV/AIDSbedingten Todesursache, sind es nach Schätzungen des RKI 1998 nur noch etwa 600 .

Von den neuen Möglichkeiten in der Therapie profitieren verschiedene Gruppen jedoch in unterschiedlichem Maße. Während die Zahl der diagnostizierten AIDS-Fälle bei homo- bzw. bisexuellen Männern um 66\% im Vergleich zu 1995 zurückging, beträgt der Rückgang bei den intravenös Drogenabhängigen $60 \%$ und bei heterosexuell Infizierten sogar "nur" $33 \%$. Vermeiden oder hinausschieben läßt sich eine AIDSErkrankung mit Hilfe neuer Therapien natürlich nur, wenn die HIV-Infektion bekannt ist und medizinische Hilfe in Anspruch genommen wird. Eine offenbar gleichbleibend große Gruppe von Personen scheint aber Infektionsrisiken zu verdrängen und Testangebote nicht wahrzunehmen: ein Indiz dafür ist die Tatsachè, $\mathrm{da} B$ die Zahl der AIDS-Patienten, die keine Angaben zum vermuteten Infektionsweg machen konnten oder wollten, gegenüber dem Jahr 1995 nahezu unverändert bleibt. Die Zahl der AIDS-Erkrankungen bei Personen, die in Deutschland leben und aus Ländern stammen, in denen sich die HIV-Infektion in der gesamten Bevölkerung stark ausgebreitet hat, nahm sogar zu.
Dic therapiebedingte Verringerung der Zahl der AIDS-Erkrankungen darf nicht mit einem Rückgang der HIV-Neuinfektionen gleichgesetzt werden. Für eine deutliche Abnahme der HIV-Neuinfektionen in den letzten Jahren gibt es auf der Basis der vorliegenden Daten bisher keinen Anhaltspunkt. Die Zahl neuer HIV-Infektionen wird vom RKI auf immer noch etwa 2000 pro Jahr geschätzt.

Durch Verbesserungen im Meldesystem liegen für das Jahr 1998 Angaben zum Infektionsweg für über $80 \%$ der gemeldeten HIV-Befunde vor. Die größte Gruppe unter den HIV-Neuinfektionen bleiben mit etwa $40 \%$ die homo- bzw. bisexuellen Männer. Der Anteil der intravenös Drogenabhängigen ist in den letzten Jahren auf $10 \%$ zurückgegangen. Dagegen mach der-Anteil auf heterosexuellem Weg Infizierten mittlerweile $16 \%$ aus. Auch der Anteil von Personen, die aus Ländern mit einer hohen HIV-Vereuchung stammen und in denen der heterosexuelle Übertragungsweg vorherrschend ist, ist auf $16 \%$ gestiegen. Für 17\% liegen keine Angaben zum Infektionsweg vor.

Abzüglich der bereits an AIDS oder vor dem Eintreten von AIDS-definierenden Erkrankungen Verstorbenen, lebten Ende 1998 in Deutschland etwa 37000 HIV-infizierte Personen. Auf Grund der heute verbesserten therapeutischen Möglichkeiten und der dadurch bedingten Abnahme der Todesfälle, wird die Zahl der zu versorgenden HIV- bzw. AIDS-Patienten bei einer stabilen Zahl von HIV-Neuinfektionen weiter leicht anwachsen. Bei einer wachsenden HIV-Prävalenz ist jedoch zu erwarten, daß ohne eine tatsächliche Heilung sowohl die Zahl der Neuerkrankungen als auch die der Todesfälle wieder ansteigen werden, da die bisherigen Therapien lediglich den Krankheitsverlauf verzögern, nicht aber ganz aufhalten können. Gleichzeitig ist davon auszugehen, daß die Zahl der zu versorgenden Patienten weiter anwächst.

\section{RKI-P}


Robert Koch-Institut legt erste Auswertungsergebnisse des Bundesgesundheitssurveys vor: Drastischer Anstieg der Zahl der Rau-
cherinnen in den neuen Bundesländern

Nachdem die Untersuchungen und Befragungen einer repräsentativen Auswahl von erwachsenen Einwohnern aus 150 verschiedenen Orten der Bundesrepublik Deutschland im Frühjahr dieses Jahres abgeschlossen waren, liegt dem Robert Koch-Institut nunmehr ein umfassender Datensatz mit anonymisierten Informationen zum Gesundheitszustand für 7.124 Männer und Frauen im Alter von 18 bis 80 Jahren vor. Damit existieren aktuelle Daten, die Aussagen ermöglichen zu zeitlichen Trends in Gesundheitszustand und Gesundheitsverhalten der deutschen Bevölkerung. Insbesondere die Entwicklung der bei der letzten Survey-Erhebung 1991/92 festgestellten Ost-West-Unterschiede, aber auch Informationen zur Inanspruchnahme von Präventionsangeboten oder zur Entwicklung gesundheitsbewußter Verhaltensweisen, sind von großem Interesse. Diese Aussagen sollen sowohl Bestandteil einer zeitnahen Gesundheitsberichterstattung als auch Produkt epidemiologischer Forschung und Grundlage gesundheitspolitischer Weichenstellungen werden.

Aus diesem Grund sehen die Mitarbeiter des Robert Koch-Instituts ihre Verantwortung darin, möglichst schnell und in geplanter Reihenfolge an die Auswertung dieses Datenschatzes zu gehen. Wegen seiner gesundheitlichen Relevanz war das Rauchen Gegenstand der ersten Auswertungen des Bundesgesundheitssurveys. Die Ergebnisse wiederum geben keinen Anlaß zu Optimismus:

Im Jahre 1998 rauchte ein Drittel der Bevölkerung im Alter von 18 bis $80 \mathrm{Jahren;} 37 \%$ der Männer und $28 \%$ der Frauen. In den jüngsten Altersgruppen sind die Raucheranteile am höchsten: $49 \%$ der männlichen 18-25jährigen und 44\% der Frauen dieser Altersgruppe rauchen.

Bei Betrachtung der-Entwicklungen in den alten und neuen Bundesländern ist folgendes zu erkennen: In den alten Bundesländern sank der Raucheranteil bei Männern um 2 Prozentpunkte auf 36\%, bei Frauen blieb er auf gleicher Höhe (28\%). In den neuen Bundesländern stieg der Anteil der Raucher bei Männern um einen Prozentpunkt auf $41 \%$. Alarmierend ist allerdings der Zuwachs ostdeutscher Raucherinnen: Während dort noch 1992 21\% der Frauen angaben zu rauchen, sind es jetzt schon 29\%. Damit liegt der Anteil rauchender Frauen in den neuen Bundesländern jetzt sogar um einen Prozentpunkt über den alten Bundesländern. „Der schon früher befürchtete weitere Anstieg der Lungenkrebs-Sterblichkeit bei Frauen könnte damit in absehbarer Zeit Realität werden", vermutet Dr. Bärbel-Maria Bellach, Leiterin der Abteilung für Epidemiologie und Gesundheitsberichterstattung am Robert Koch-Institut.
Auch zur Rauchintensität liegen Zahlen aus dem Bundesgesundheitssurvey vor. $46 \%$ der männlichen Raucher konsumieren 20 und mehr Zigaretten täglich und sind damit als starke Raucher einzustufen. Bei den Frauen beträgt dieser Anteil 31\%. Beim Vergleich alter und neuer Bundesländer liegt der Anteil starker Raucher im Westen höher: bei Männern um 7 Prozentpunkte $(47 / 40 \%)$, bei Frauen ist er doppelt so groß (34/17\%).

Allerdings rauchen nicht alle Raucher gern. Rund ein Drittel von ihnen, Männer wie Frauen, in alten wie in neuen Bundesländern, hat während des letzten Jahres mindestens einen Versuch unternommen, mit dem Rauchen aufzuhören.

\section{RKI-P}

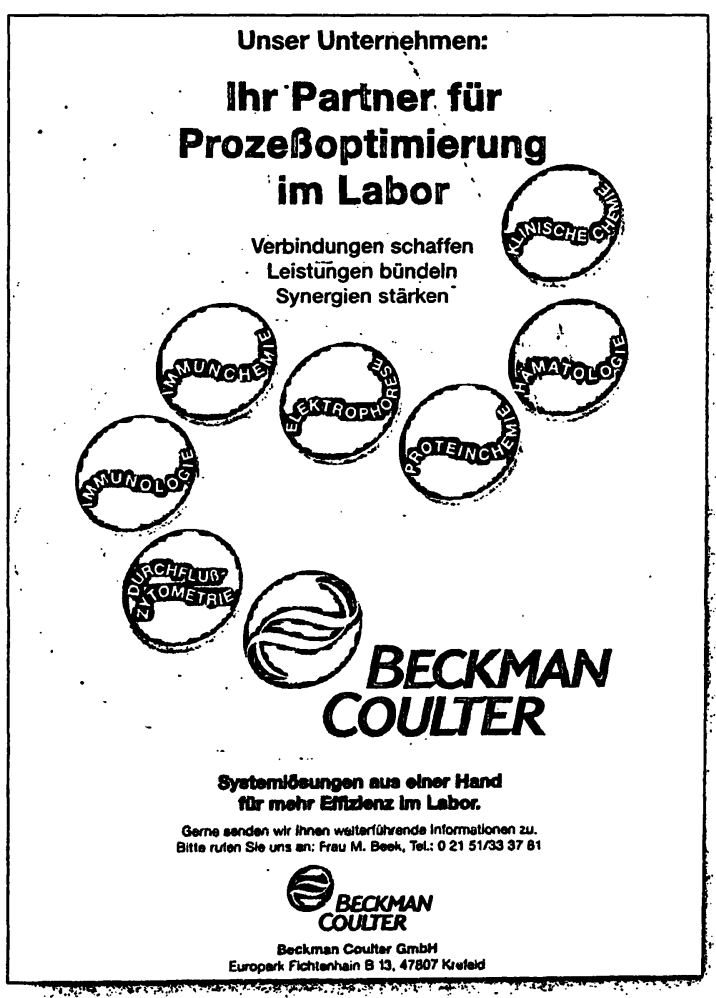

